\title{
Fibro Adipose Vascular Anomaly: A Rare and Often Misdiagnosed Entity
}

\author{
Pushpinder S. Khera ${ }^{1}$ Pawan K. Garg ${ }^{1}$ Santhosh Babu ${ }^{1} \quad$ Poonam Elhence $^{2}$ Sarbesh Tiwari ${ }^{1}$ \\ Shyamkumar $\mathrm{NK}^{3}$
}

1 Department of Diagnostic and Interventional Radiology, All India
Institute of Medical Sciences, Jodhpur, Rajasthan, India
2Department of Pathology, All India Institute of Medical Sciences,
Jodhpur, Rajasthan, India
${ }^{3}$ Department of Radiology, Christian Medical College and Hospital,
Vellore, Tamil Nadu, India Indian J Radiol Imaging 2021;31:776-781.
Address for correspondence Pawan Kumar Garg, MD, Department of Diagnostic and Interventional Radiology, AlIMS, Jodhpur, Rajasthan 342005, India (e-mail: drgargpawan@gmail.com).

\author{
Abstract \\ Keywords \\ - fibro adipose vascular \\ anomaly \\ - vascular \\ malformation \\ - venous malformation
}

Fibro adipose vascular anomaly (FAVA) is a rare type of vascular malformation with distinct clinical features. The authors here discussed the clinical, imaging, differential diagnosis, histopathological features, and treatment options of FAVA along with an illustrative case. It is important to know about this uncommon entity as this can be misdiagnosed due to the overlapping clinical features with other common entities. It is a benign condition with no proven malignant potential. There are no guidelines regarding the best treatment option.

\section{Introduction}

Fibro adipose vascular anomaly (FAVA) is a type of vascular malformation which is classified under the International Society for the Study of Vascular Anomalies (ISSVA) in the provisionally unclassified vascular malformation group., ${ }^{1,2}$ FAVA tends to occur more in young females and lower extremities. Clinical, imaging findings, and histopathological correlations are needed for better understanding of the disease and to differentiate it from other vascular malformations. No single treatment is available for FAVA. A combination of sclerotherapy, intralesional steroids, cryotherapy, or ablation therapy can be tried. If there is restriction in movement surgical cut down/resection can be done.

\section{Case Report}

A 14-year-old female referred from the orthopaedics department for the evaluation of swelling over the posterior aspect of the left lower limb in suspicion of venous malformation (VM). The patient had swelling over the calf and dorsal aspect of the left foot for the past 10 years which was insidious in onset, gradually progressive, and associated with severe constant pain at the local site with restriction in movement. No history of trauma, fever, or similar family history was observed. There was past history of sclerotherapy and Achilles tendon release 2 to 3 years back, however, there was no much improvement. Routine blood investigations including coagulation profile, erythrocyte sedimentation rate, and C-reactive protein were within normal limits. On clinical examination, the left lower limb was thinner compared with the right ( - Fig. 1). There were multiple areas of soft swelling over the posterior aspect of the left leg and lateral aspect of the dorsum of the left foot. Palpation of the local site had tenderness and mild increase in temperature. There were restricted dorsiflexion movements and the tendency of toe walking. Motor power in the lower limb and the overlying skin was normal. The scar mark of the tendon release
DOI https://doi.org/ 10.1055/s-0041-1736399. ISSN 0971-3026.

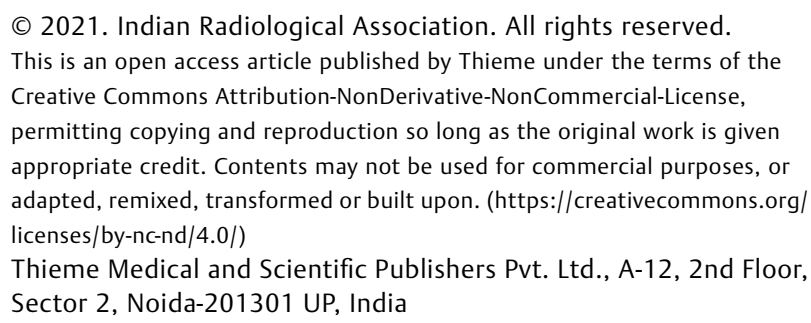




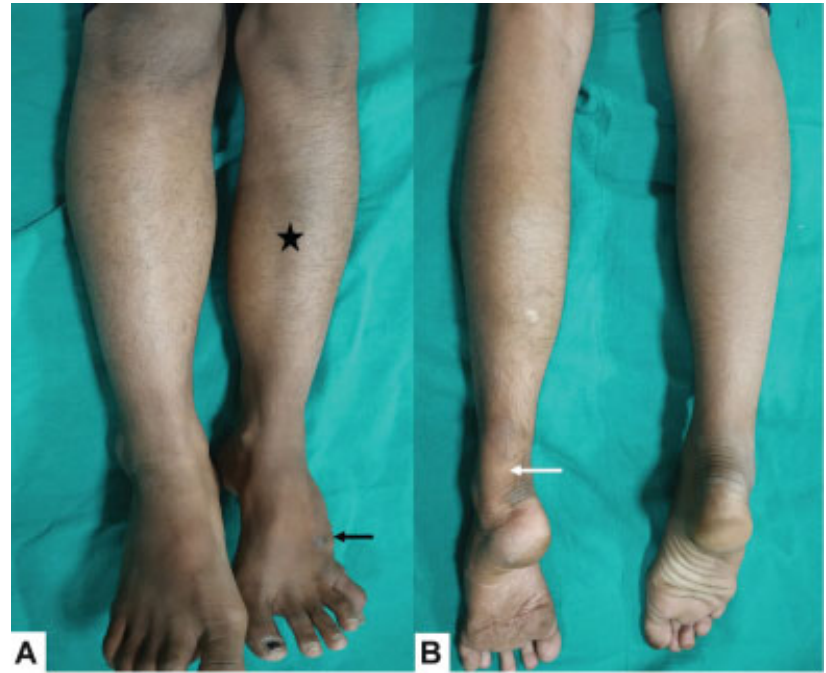

Fig. 1 Clinical image of patient anterior (A) and posterior view (B), showing relative atrophy of left leg (asterisk). The lesion is predominantly involving the posterior aspect of the leg, also extending to the dorsum of the foot (black arrow). Scar of previous tendo-achilles release is seen (white arrow).

procedure was also noted along the posterior part of the ankle.

The patient was extensively investigated with all imaging modalities during the course of her disease without a definitive diagnosis. Radiograph of left leg revealed few abnormal soft tissue radiopacity over the posterior aspect with dense amorphous calcification (-Fig. 2A, B). Mild thinning of the fibula is seen due to long-standing pressure remodeling from the swelling. Ultrasound of the local site showed heterogenous predominantly echogenic well-circumscribed noncompressible soft tissue lesions in the intramuscular and subcutaneous plane in the posterior aspect of the left leg (-Fig. 2C, D). There were few echogenic foci noted giving posterior acoustic shadowing suggestive of calcifications (-Fig. 2E). Few anechoic serpiginous channels were also noted with minimal color filling and low-velocity venous type of waveform on spectral imaging (-Fig. 2F).

Non-contrast computed tomography (NCCT) revealed well-defined lobulated heterogenous hyperdense mass lesions in the intramuscular and subcutaneous plane. Areas of fat attenuation replacing the lateral and medial head of gastrocnemius, soleus, and plantaris muscles were seen. Multiple clustered areas of heterogenous calcification were seen within the hyperdense lesions ( - Fig. 3A, B).

Magnetic resonance imaging (MRI) of the left lower limb showed well-defined lobulated predominantly T2W and short tau inversion recovery (STIR) hyperintense lesions in the subcutaneous and intramuscular plane. There was fatty replacement of the lateral and medial head of gastrocnemius, soleus, and plantaris muscles. Multiple hypointense areas were also noted within the lesion corresponding to calcifications. There are grouped muscle atrophy of the posterior compartment as compared with the right side. On post gadolinium, T1-weighted sequence dilated dysplastic venous channels were seen in the lesion. The lesions showed moderate enhancement in the late arterial phase which was persistent in late venous phase too ( $\mathbf{- F i g . ~ 3 C - F ) . ~ B a s e d ~ o n ~ t h e ~ c l i n i c a l ~ a n d ~ i m a g i n g ~ f e a t u r e s , ~}$ FAVA was suggested.

The ultrasound-guided biopsy was performed using an 18-gauge needle. Histopathological examination showed fibro-collagenous tissue cores with fibro adipose tissue and containing several large and few small-caliber vessels (-Fig. 4). Anastomosing retiform to ectatic vascular spaces

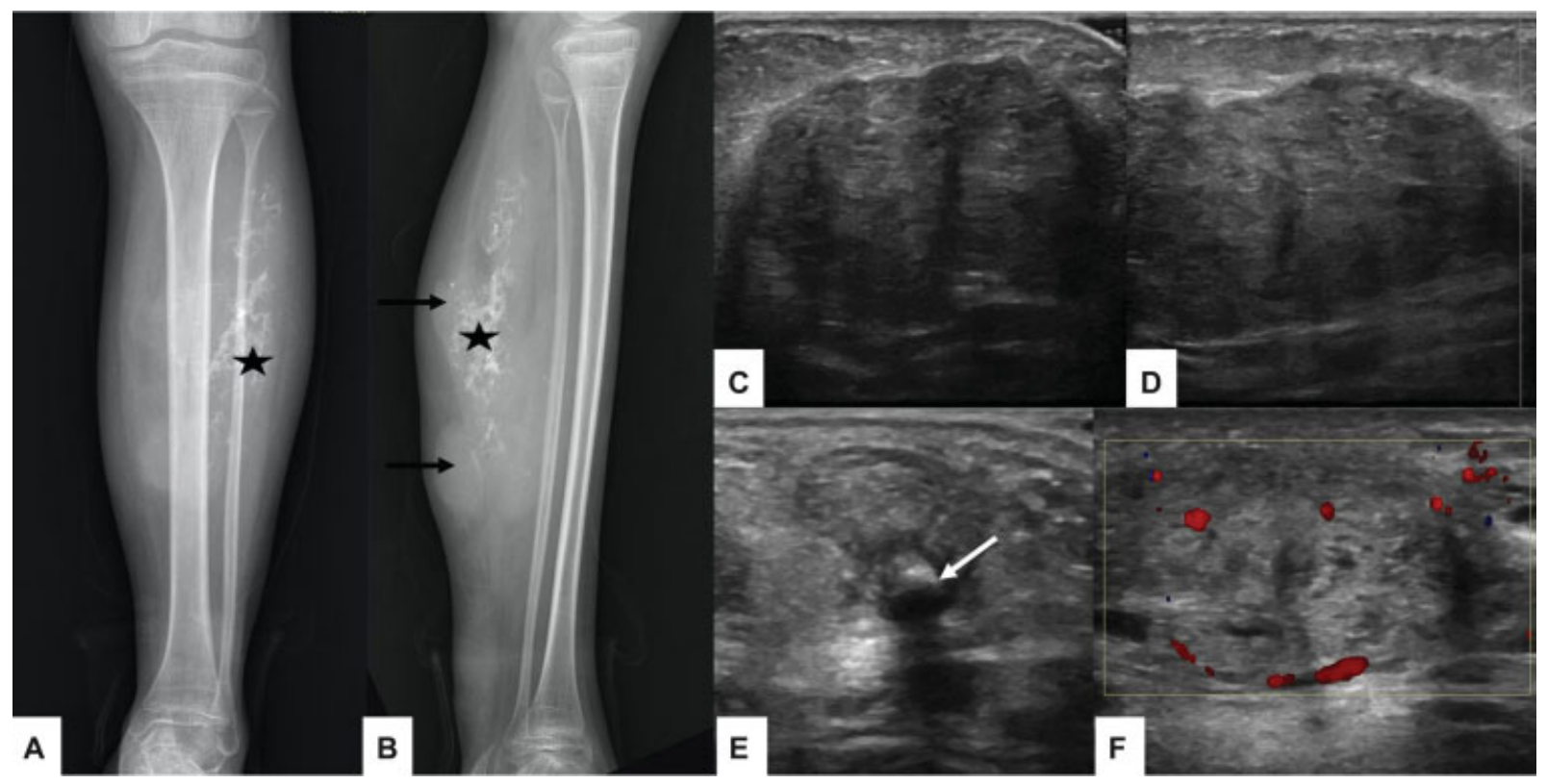

Fig. 2 Radiograph of left leg anteroposterior (A) and lateral (B) view showing lobulated soft tissue lesion (black arrow) in the calf region with dense amorphous calcification (asterisk). Thinning and scalloping of fibula without obvious bony destruction is seen. Ultrasound images (C-E) showing lobulated predominantly echogenic lesions with no compressible venous spaces. Presence of calcification (white arrow) with posterior acoustic shadowing seen within the lesion. Color Doppler (F) image showing minimal venous flow. 


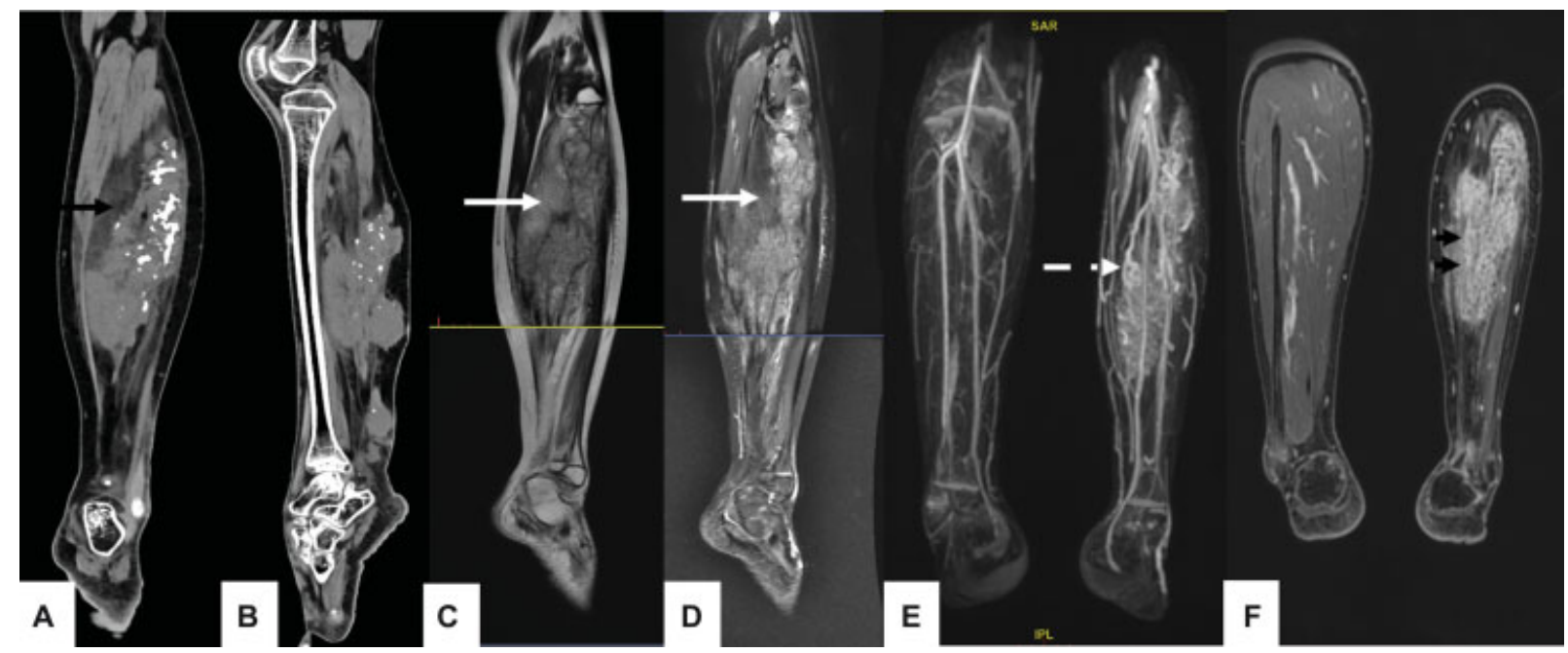

Fig. 3 Computed tomography (CT) scan in the coronal (A) and sagittal (B) plane of leg showing a lobulated isodense lesion in the leg with the presence of dense calcification and fat (black arrow) within the lesion. MRI of leg T2 (C) and short tau inversion recovery (STIR) (D) sequence sagittal images showing predominantly hyperintense lesion with fatty component (white arrow) suppressed on STIR. Dynamic contrast (E) in coronal, maximum intensity projection (MIP) image showing dysplastic venous channels (dashed arrow). Post-contrast images (F) showing homogenous enhancement in the venous phase (black arrowhead).

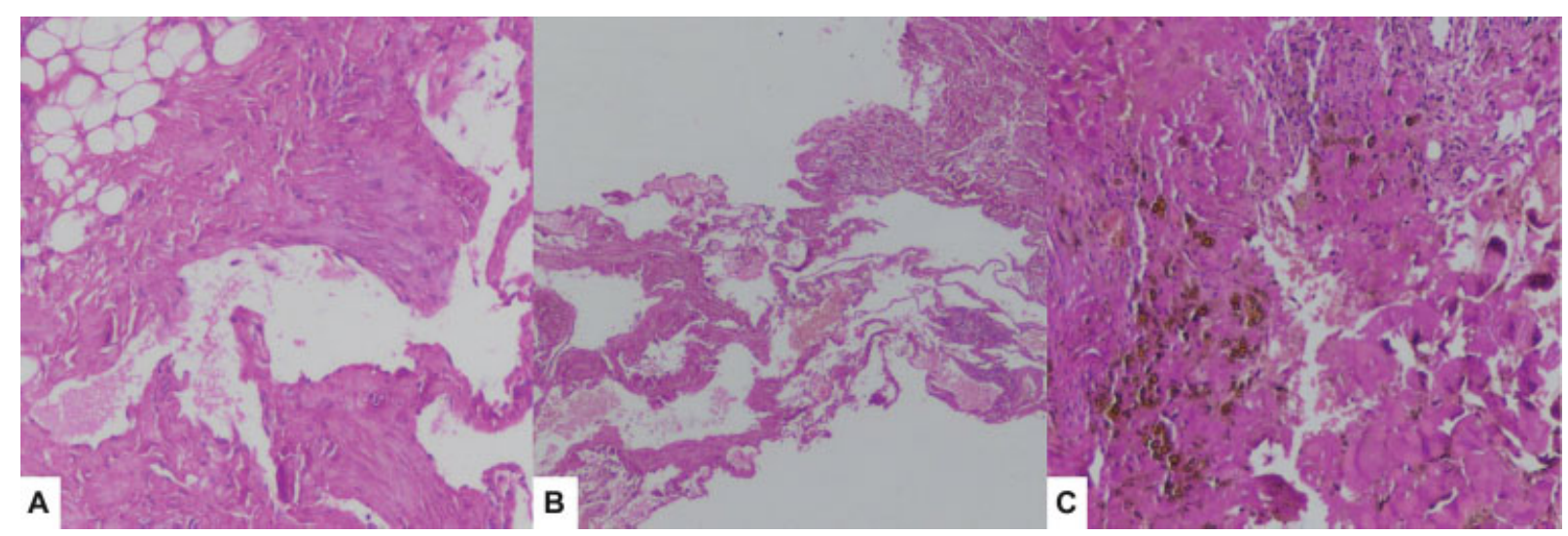

Fig. 4 Histopathology images with Hematoxylin and Eosin (H\&E) stain. (A) Showing multiple thin-walled ectatic vessels with mature adipocytic tissue. (B) Tissue core containing several anastomosed thin-walled ectatic congested vessels. (C) Core with coarse golden-brown hemosiderin pigment, few vessels, and skeletal muscle fascicles.

were lined by flattened endothelium. Aggregates of lymphocytes, perivascular mononuclear cell infiltrate, and hemosiderin pigment were noted. The final diagnosis of FAVA was confirmed.

The patient was explained about the disease and kept on regular follow-up. She was advised for symptomatic treatment in the form of local alcohol injection, cryotherapy, and surgical resection.

\section{Discussion}

Alomari et al in 2014, first time described a clinical condition named FAVA, with distinct clinical, radiological, and histopathologic features. FAVA consist of abnormal fibrofatty infiltration of muscles, venous abnormality in the form of phlebectasia, contracture of the affected extremity, and continuous pain. ${ }^{3,4}$ Historically many reports mentioned similar pathological lesions termed as intramuscular hem- angioma causing contracture, leading to toe walking and equinus deformity. ${ }^{5-7}$

International Society for the Study of Vascular Anomalies (ISSVA) classified vascular malformation into vascular tumors (neoplastic), vascular malformations (non-neoplastic), and unclassified anomalies. ${ }^{1}$ In the 2018 revision of ISSVA classification, FAVA was first time included in the provisionally unclassified vascular anomalies category. This category also includes other conditions like intramuscular hemangioma, angiokeratoma sinusoidal hemangioma, and acral arteriovenous tumor, etc. ${ }^{1,2}$

Various published literature on FAVA with patient's age, sex, site of involvement, symptoms, referring diagnosis, and treatment are compiled in $\mathbf{- T a b l e} \mathbf{1}$.

FAVA is usually sporadic and most commonly caused by somatic mutation involving PIKC3A (phosphatidylinositol-4,5bisphosphate 3-kinase) gene. The same gene is seen in most cases of isolated lymphatic and veno-lymphatic vascular 


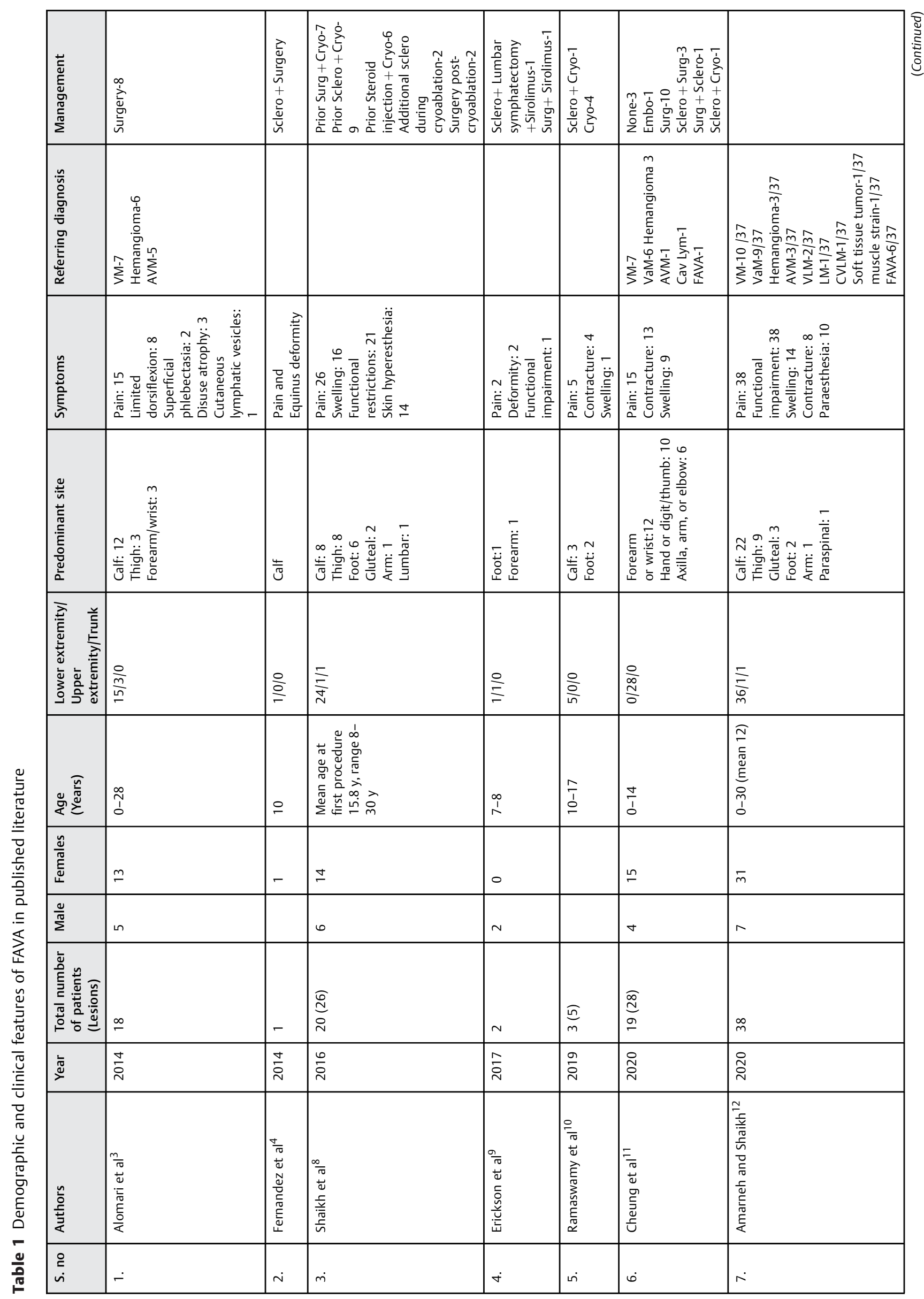




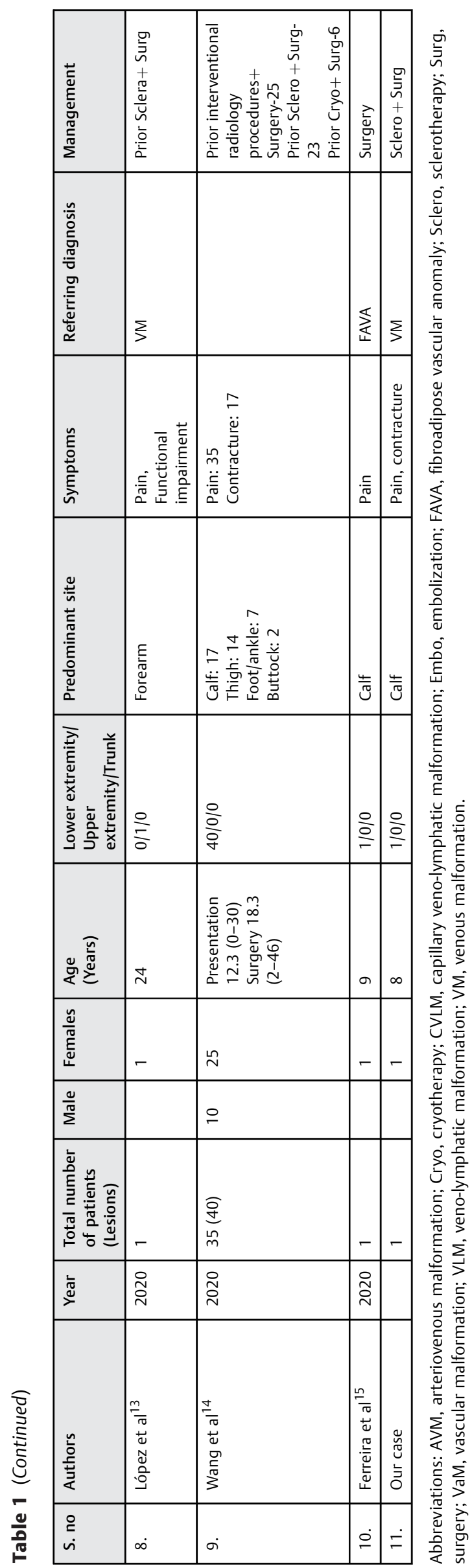

malformations. Other pathology caused by the same genetic mutation includes Klippel-Trenaunay syndrome, megalencephaly-capillary malformation-polymicrogyria (MCAP), and CLOVES (congenital lipomatous overgrowth, vascular malformations, epidermal nevi, and skeletal anomalies) syndrome. ${ }^{12,16,17}$

FAVA usually occurs in young adults with age group between 1 and 30 years. It is more common in females with male to female ratio of 1:4. Lower extremities are more commonly involved than upper extremities. Calf muscles are most commonly involved followed by the thigh. ${ }^{3,12}$ Since FAVA is a new clinical entity and has overlapping features with other conditions like VM, vascular malformation, intramuscular hemangioma, and soft tissue tumor, diagnosis is often delayed and missed. ${ }^{12}$

FAVA is a distinct entity characterized by abnormal fibrofatty masses and infiltration in intramuscular as well as subcutaneous plane. There is presence of abnormal venous channels in form of phlebectasia within masses. The fibrotic process leads to the contracture of involved muscles resulting in restriction of movement. Since calf and gastrocnemius are most commonly involved region the fibrotic process leads to equinus deformity and toe walking. ${ }^{3,4}$ The lesion is also associated with continuous pain which is multifactorial; caused by muscular contracture, neurogenic infiltration, and thrombophlebitis of VM or phlebectasia. ${ }^{3,4,12}$

FAVA can be divided into focal mass-like lesion, focal infiltrative, or diffuse infiltrative type. ${ }^{12}$ The patient usually presents with long-standing soft non-compressible swelling with restricted movement due to contracture and constant pain. There can be associated skin changes in some cases like ulcers, venous engorgements, lymphatic vesicles, and skin hypopigmentation.

Imaging plays an important role in the diagnosis of FAVA and to differentiate it from other clinical conditions. Ultrasound usually shows heterogeneous echogenic masses due to fibrofatty proliferation within the muscles and subcutaneous plane. In comparison with VM, FAVA is predominantly solid with minimal or no compressible spaces. On Doppler, if venous channels are patent then venous flow can be seen. $\mathrm{X}$-ray or CT scan can show soft tissue intramuscular isodense masses along with the presence of dystrophic calcification in long-standing cases.

MRI is imaging modality useful in the differentiation of FAVA from other lesions. MRI shows well-defined intramuscular masses which are hyperintense on T1, T2W due to presence of fatty component, however, less hyperintense on $\mathrm{T} 2 \mathrm{~W}$ images in comparison to $\mathrm{VM}$ due to the presence of fluid in later. Also, on STIR sequence fatty component shows signal suppression. On post gadolinium sequence heterogenous enhancement is seen mainly in late arterial or venous phases. Direct puncture and intralesional venography may demonstrate the network of anomalous dysplastic veins.

Despite characteristic clinical features and imaging features, histopathological confirmation is required because of the rarity of the lesion. Histopathologically FAVA shows the presence of abnormal fibrous and fatty tissue in atrophied skeletal muscles with abnormal ectatic venous channels and lymphoplasmacytic/lymphoid aggregates. ${ }^{3,12}$ 
There is no single definitive treatment of FAVA. The imageguided treatment option is local sclerotherapy of venous components. Intralesional steroid and alcohol injection, local cryotherapy, and nerve blocks for pain relief. Surgical excision is an option with procedure-related morbidity as most of these lesions are intramuscular. Debulking of mass with contracture release to improve deformity, movement restriction, and pain relief in selective cases. Sirolimus is a drug also used in the treatment of FAVA but not approved in less than 18 years. To summarize, treatment is mostly symptomatic to address patient predominant clinical problems. ${ }^{8,9,14}$

\section{Conclusion}

FAVA is an uncommon but distinct clinical entity with typical presentation in young females and mostly involves lower limb. The classical presentation is long-standing swelling with constant pain, contracture, restricted movement, and deformity. However, because of the rarity of cases, recently described condition, overlapping features with other common entities, and lesser awareness, it is often misdiagnosed. The multimodality approach and patient education play a major role in management.

\section{Declaration of Patient Consent}

The authors certify that they have obtained all appropriate patient consent forms. In the form the patient(s) has/have given his/her/their consent for his/her/their images and other clinical information to be reported in the journal. The patients understand that their names and initials will not be published, and due efforts will be made to conceal their identity, but anonymity cannot be guaranteed.

\section{Financial Support and Sponsorship \\ None.}

\section{Source(s) of Support}

None.

\author{
Presentation at a Meeting \\ None. \\ Organization \\ Place \\ Date
}

Conflict of Interest

None declared.

\section{References}

1 Merrow AC, Gupta A, Patel MN, Adams DM. 2014 revised classification of vascular lesions from the International Society for the study of vascular anomalies: Radiologic-pathologic update. Radiographics 2016;36(05):1494-1516

2 Available at. https://www.issva.org/UserFiles/file/ISSVA- Classification-2018.pdf

3 Alomari AI, Spencer SA, Arnold RW, et al. Fibro-adipose vascular anomaly: clinical-radiologic-pathologic features of a newly delineated disorder of the extremity. J Pediatr Orthop 2014;34(01): 109-117

4 Fernandez-Pineda I, Marcilla D, Downey-Carmona FJ, Roldan S, Ortega-Laureano L, Bernabeu-Wittel J. Lower extremity fibroadipose vascular anomaly (FAVA): a new case of a newly delineated disorder. Ann Vasc Dis 2014;7(03):316-319

5 Klemme WR, James P, Skinner SR. Latent onset unilateral toewalking secondary to hemangioma of the gastrocnemius. J Pediatr Orthop 1994;14(06):773-775

6 Umehara F, Matsuura E, Kitajima S, Osame M. Unilateral toewalking secondary to intramuscular hemangioma in the gastrocnemius. Neurology 2005;65(07):E15

7 Clarke RC, Wenger RJJ. Equinus deformity and haemangioma of calf muscle. BMJ 1975;3(5978)

8 Shaikh R, Alomari AI, Kerr CL, Miller P, Spencer SA. Cryoablation in fibro-adipose vascular anomaly (FAVA): a minimally invasive treatment option. Pediatr Radiol 2016;46(08):1179-1186

9 Erickson J, McAuliffe W, Blennerhassett L, Halbert A. Fibroadipose vascular anomaly treated with sirolimus: successful outcome in two patients. Pediatr Dermatol 2017;34(06):e317-e320

10 Ramaswamy RS, Tiwari T, Darcy MD, et al. Cryoablation of lowflow vascular malformations. Diagn Interv Radiol 2019;25(03): 225-230

11 Cheung K, Taghinia AH, Sood RF, et al. Fibroadipose vascular anomaly in the upper extremity: a distinct entity with characteristic clinical, radiological, and histopathological findings. J Hand Surg Am 2020;45(01):68.e1-68.e13

12 Amarneh M, Shaikh R. Clinical and imaging features in fibroadipose vascular anomaly (FAVA). Pediatr Radiol 2020;50(03): 380-387

13 López S, Ibarra A, Baselga E, et al. Fibro-adipose vascular malformation (FAVA) in forearm: a successful surgical approach and literature review. Eur J Plast Surg 2020;43:495-500

14 Wang KK, Glenn RL, Adams DM, et al. Surgical management of fibroadipose vascular anomaly of the lower extremities. J Pediatr Orthop 2020;40(03):e227-e236

15 Ferreira J, López Gutiérrez JC, Carneiro A, et al. Lower extremity fibro-adipose vascular anomaly-case report. Ann Vasc Surg 2020;66:671.e15-671.e18

16 Luks VL, Kamitaki N, Vivero MP, et al. Lymphatic and other vascular malformative/overgrowth disorders are caused by somatic mutations in PIK3CA. J Pediatr 2015;166(04): 1048-54.e1, 5

17 Keppler-Noreuil KM, Rios JJ, Parker VER, et al. PIK3CA-related overgrowth spectrum (PROS): diagnostic and testing eligibility criteria, differential diagnosis, and evaluation. Am J Med Genet A 2015; $167 \mathrm{~A}(02): 287-295$ 